\title{
Association between Bladder Outlet Obstruction and Bladder Cancer in Patients with Aging Male
}

\author{
Yu-Hsiang Lin $1,2,3,+\oplus$, Chen-Pang Hou ${ }^{1,2,3,+}$, Horng-Heng Juang ${ }^{1,2,4} \oplus$, Phei-Lang Chang ${ }^{1,2}$, \\ Tien-Hsing Chen ${ }^{2,5}$, Chien-Lun Chen ${ }^{1,2}$ and Ke-Hung Tsui ${ }^{1,2, *(D)}$ \\ 1 Department of Urology, Chang Gung Memorial Hospital at linkou, Taoyuan 333, Taiwan; \\ linyh@cgmh.org.tw (Y.-H.L.); glucose1979@gmail.com (C.-P.H.); hhj143@mail.cgu.edu.tw (H.-H.J.); \\ henryc@cgmh.org.tw (P.-L.C.); clc2679@cgmh.org.tw (C-L.C.) \\ 2 School of Medicine, Chang Gung University, Taoyuan 333, Taiwan; skyheart0826@gmail.com \\ 3 Graduate Institute of Clinical Medical Sciences, College of Medicine, Chang Gung University, \\ Taoyuan 333, Taiwan \\ 4 Department of Anatomy, School of Medicine, Chang Gung University, Kwei-shan, Tao-Yuan 333, \\ Taoyuan, Taiwan \\ 5 Department of Cardiology, Chang Gung Memorial Hospital at Keelung, Biostatiscal Consultation Center of \\ Chang Gung Memorial hospital, Community Medicine Research Center, Keelung, Keelung 204, Taiwan \\ * Correspondence: khtsui@yahoo.com \\ + These authors contribute equally to this paper.
}

Received: 14 August 2019; Accepted: 23 September 2019; Published: 27 September 2019

check for updates

\begin{abstract}
The associations between the treatment outcomes of benign prostatic hyperplasia/benign prostatic obstruction and lifelong health status, including urologic cancer incidence as well as geriatric adverse events (AEs), are unknown. This retrospective cohort study analyzed claims data collected during the period of 1997-2012 from Taiwan's Longitudinal Health Insurance Database 2000. Patients who received transurethral resection of the prostate (TURP) were prioritized, and the remaining patients who were prescribed alpha-blockers were, subsequently, identified. Patients in the TURP and medication-only groups were further divided into two groups, according to the presence or absence of AEs during the first six-month follow-up. Outcomes of primary interest were all-cause mortality, occurrence of prostate cancer, transurethral resection of the bladder tumor, and radical cystectomy for bladder cancer. Compared with patients in the AE-free TURP group, those in the TURP with AEs had a higher risk of lifelong bladder cancer (subdistribution hazard ratio: $2.3,95 \%$ confidence interval (CI): 1.56-3.39), whereas the risk of prostate cancer was comparable between the two groups (SHR: 1.2, 95\% CI: 0.83-1.74). In the medication cohorts, patients undergoing alpha-blocker treatment who had AEs had a higher risk of all-cause mortality (hazard ratio: 1.63, 95\% CI: 1.49-1.78) and a higher risk of lifelong bladder cancer (SHR: 2.72, 95\% CI: 1.99-3.71) when compared with those without AE. Our study reveals that unfavorable treatment outcomes of benign prostate hyperplasia, whether caused by medication or surgical treatment, are associated with a higher incidence of bladder cancer. Unfavorable outcomes of surgical treatment are associated with higher risk of geriatric AEs, and unfavorable outcomes of medication treatment are associated with a higher risk of all-cause mortality.
\end{abstract}

Keywords: benign prostate hyperplasia; bladder outlet obstruction; diabetes mellitus; prostatectomy; urinary tract infection; lower urinary tract symptoms

\section{Introduction}

Benign prostatic hyperplasia (BPH), which is a key cause of lower urinary tract symptoms (LUTS) in older men, affects approximately 210 million men worldwide [1]. A study revealed that $50 \%$ of men developed pathological BPH at the age of 51-60 years [2]. Moreover, BPH/LUTS prevalence is expected 
to increase sharply in the coming decades [3]. The sequelae of BPH include decreased urinary flow and progression of voiding and storage symptoms, which eventually results in acute or chronic urinary retention (UR) [4]. BPH with moderate-to-severe LUTS considerably affects all aspects of the quality of life of men as they age [5]. A 50-year-old from the United States has an estimated risk of approximately $40 \%$ of undergoing therapeutic intervention (surgical or medical treatment) at some point during his lifetime [6]. Accordingly, billions of US dollars are spent annually to treat BPH/LUTS [7].

Both alpha-1 blockers and transurethral resection of the prostate (TURP) achieve favorable treatment outcomes in most patients with benign prostatic obstruction (BPO) [8]. Alpha-1 blockers are used for first-line treatment of BPO in men with LUTS [9]. In patients with unsatisfactory response to medication, TURP remains the dominant and definitive treatment for LUTS caused by BPH [10]. Some studies have also reported that TURP achieves favorable outcomes even for BPH patients with comorbidities such as UR, type 2 diabetes, and stroke [11-13]. Nevertheless, to the best of our knowledge, no studies have addressed the long-term sequelae for patients with "unfavorable" outcomes from either medical or surgical treatment. Therefore, we used Taiwan National Health Insurance Research Database (NHIRD) data to conduct a nationwide observational cohort study for investigating the correlation between the treatment outcomes of BPH/LUTS and lifelong health status, including urologic cancer incidence and geriatric adverse events.

\section{Methods}

\subsection{Data Source}

In this study, we used data from the Longitudinal Health Insurance Database 2000 (LHID2000), which is a subset of National Health Insurance research database (NHIRD.). LHID2000 contains the claims data of beneficiaries in the National Health Insurance (NHI) program of Taiwan, including details such as dates of inpatient and outpatient services, diagnoses, prescriptions, examinations, operations, and expenditures [14]. LHID2000 includes the claims data of 1,000,000 individuals randomly sampled from all NHI enrollees (a total of 23.75 million people) in 2000. The demographic characteristics (i.e., age and sex) between the populations derived from the NHIRD and LHID2000 are comparable. This study was approved by the Institutional Review Board of Chang Gung Memorial Hospital (Linkou Branch) (CMRP104-7810B).

\subsection{Patient Identification and Definition of Exposure}

We identified patients who had at least two outpatient diagnoses of BPH (International Classification of Diseases, Ninth Revision, Clinical Modification (ICD-9-CM) code: 600.xx) between 1 January, 1997 and 31 December, 2012. We first identified patients who received TURP between 1997 and 2012 by using the Taiwan NHI reimbursement codes of inpatient claims. The remaining patients who were prescribed alpha blockers for 90 days or longer within six months of the date of initial BPH diagnosis were classified as the medication-only group. For the TURP group, the index date was the discharge date following admission for TURP. For the medication-only group, the index date was the date of the initial prescription of alpha blockers for BPH.

Patients who met the following criteria were excluded: (1) age $<50$ years, (2) a diagnosis of prostate cancer or bladder cancer before the index date, (3) episodes of prostate cancer, bladder cancer, or all-cause mortality within six months post treatment, and (4) follow-up of $<6$ months. Lastly, 6254 patients were included in the TURP group and 47,965 patients were included in the medication-only group.

The TURP and medication-only groups were further divided into two groups each, according to the presence or absence of adverse events (AEs) during the first six-month follow-up period. An AE was defined as follows: the occurrence of acute UR, urinary tract infection (UTI) with prescription of antibiotics, hematuria requiring endoscopic treatment, or bladder stone formation 31-180 days after the index date. Records of UTIs and bladder stone formation were extracted from the outpatient, emergency 
room, or inpatient claim data according to diagnostic codes (plus antibiotics) and Taiwan National Health Insurance (NHI) reimbursement codes, respectively. Urine retention (UR) was recorded only in outpatient and emergency room visits. The flow for patient selection is illustrated in Figure 1.

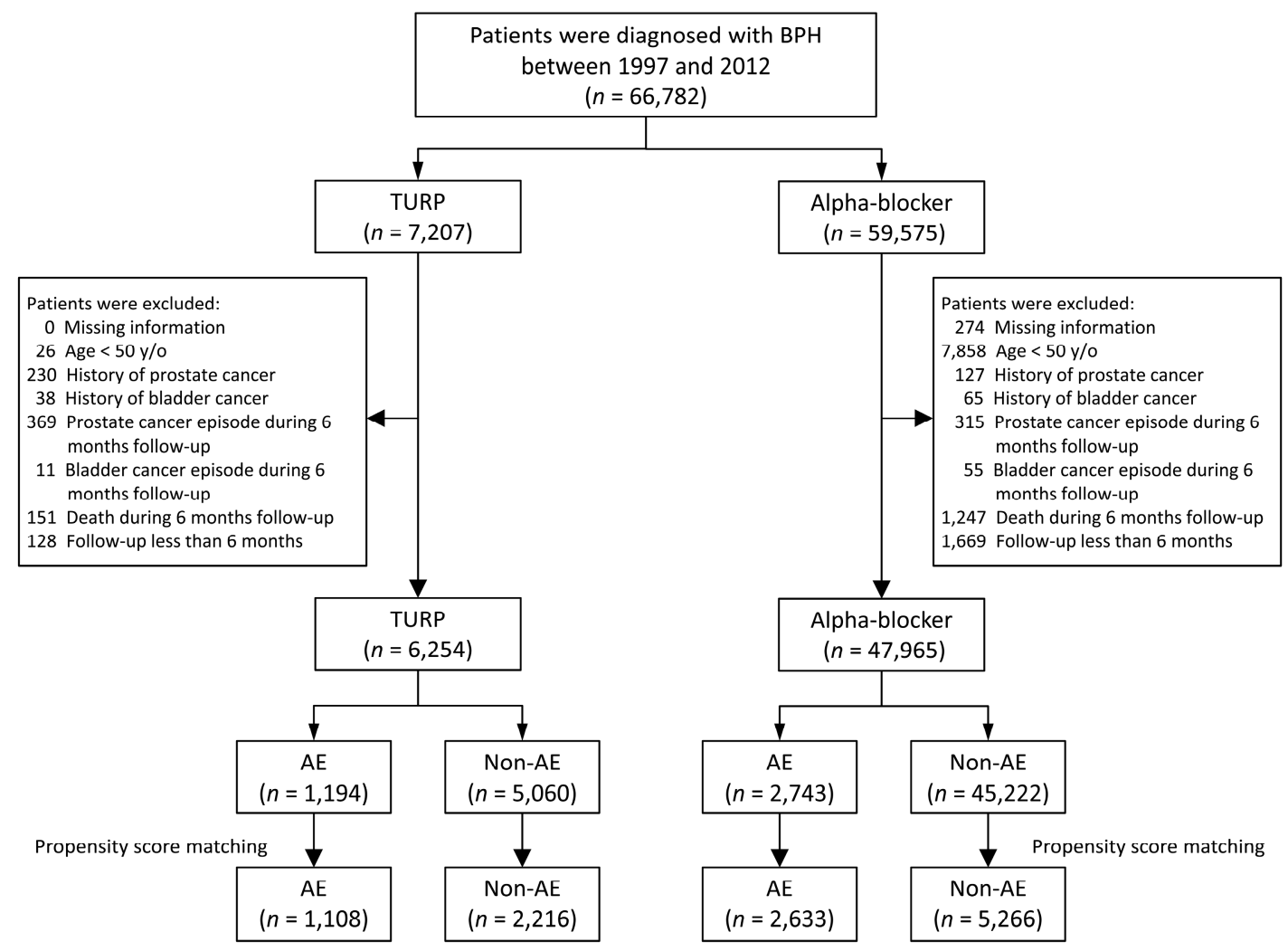

Figure 1. Patient selection.

\subsection{Covariates}

The covariates were age, comorbidities, Charlson comorbidity index score [15], tissue ablation, number of outpatient visits in the previous year, admission and AEs in the previous three years, use of statin or nonsteroidal anti-inflammatory drugs in the previous year, and use of overactive bladder drugs or alpha blockers. A comorbidity was defined as two outpatient diagnoses or one inpatient diagnosis in the previous year. Many diagnoses of these diseases in the NHIRD have been validated in relevant studies [16]. The detailed International Classification of Disease, Ninth Modification (ICD-9-CM) diagnostic codes are listed in Table 1. Medications were identified from the claim data of outpatient visits or records of pharmacy refills.

Table 1. ICD-9-CM codes used in the current study.

\begin{tabular}{cc}
\hline Variable & Code \\
\hline Benign prostatic hyperplasia & $600 . x x, \mathrm{~A} 360$ \\
\hline Prostate cancer & $185 . x x$ (Catastrophic illness certificate) \\
\hline Bladder cancer & $188.9 x$ (Catastrophic illness certificate) \\
\hline Diabetes mellitus & $250 . x x$ \\
\hline Hypertension & $401 . x x-405 . x x$ \\
\hline Hyperlipidemia & $272 . x x$ \\
\hline Chronic obstructive pulmonary disease & $491 . x x, 492 . x x, 496 . x x$ \\
\hline Parkinsonism & $332 . x x$ \\
\hline
\end{tabular}


Table 1. Cont.

\begin{tabular}{|c|c|}
\hline Variable & Code \\
\hline Chronic kidney disease & $\begin{array}{l}\text { 580.xx-589.xx, 403.xx-404.xx, 016.0x, 095.4x, 236.9x, 250.4x, 274.1x, 442.1x, } \\
447.3 x, 440.1 x, 572.4 x, 642.1 x, 646.2 x, 753.1 x, 283.11,403.01,404.02,446.21\end{array}$ \\
\hline Ischemic heart disease & 410.xx-414.xx \\
\hline Stroke & 430.xx-434.xx \\
\hline Heart failure & 428.xx \\
\hline Alcoholism & V113, 291.xx, 305.0x, 357.5, 425.5, 303.xx, 571.0, 571.1, 571.2, 571.3, 980.0 \\
\hline Drug abuse & 303.xx-305.xx \\
\hline Urinary tract infection & $599.0 x, 595.0 x$ \\
\hline Hemorrhoids & 455.xx \\
\hline Acute myocardial infarction & 410.xx \\
\hline Hip fracture & 820.xx \\
\hline Spine fracture & 805.xx, 806.xx \\
\hline
\end{tabular}

\subsection{Outcome Detection}

Outcomes of primary interest were all-cause mortality, occurrence of prostate cancer, transurethral resection of the bladder tumor (TUR-BT), and radical cystectomy for treating bladder cancer. All-cause mortality was defined as withdrawal from the NHI program [17]. The occurrence of prostate cancer was verified by the approval to possess a catastrophic illness certificate. Definitions of prostate cancer have been widely reported in NHIRD studies [18]. TUR-BT and radical cystectomy operations were identified, according to the Taiwan NHI reimbursement codes of inpatient claim data. Secondary outcomes were long-term use of Benign Prostate Hypertrophy (BPH) medications (according to pharmacy refill records), recurrent adverse events (AEs)AEs, inguinal hernia, hemorrhoids, stroke, acute myocardial infarction, hip fracture, and spine fracture during the first three-year follow-up period. Each patient was followed from the index date to the date of event occurrence, date of death, or 31 December, 2012, whichever came first.

\subsection{Statistics}

To mitigate confounding factors due to treatment selection bias in this observational study, we adopted a propensity score matching method. The propensity score was the predicted probability of being in the AE group during the first 6-month follow-up, according to the values of covariates obtained using a logistic regression. Table 2 lists the variables selected to calculate the propensity score, where the follow-up year was replaced by the index date (Table 2). Each patient in the AE group was matched with two corresponding patients in the non-AE group. The matching was processed using a greedy nearest neighbor algorithm with a caliper that was 0.2 -times the value of the standard deviation of the logit of the propensity score by applying a random matching order without replacement. The quality of matching was assessed using the absolute value of the standardized difference (STD) between the groups, where a value $<0.1$ was considered a negligible difference. 
Table 2. Baseline characteristics of patients with BPH who received TURP grouped according to the presence or absence of AEs during the six-month follow-up period.

\begin{tabular}{|c|c|c|c|c|c|c|}
\hline \multirow{2}{*}{ Variable } & \multicolumn{3}{|c|}{ Before Matching } & \multicolumn{3}{|c|}{ After Matching } \\
\hline & $\mathrm{AE}(n=1194)$ & Non-AE $(n=5060)$ & STD & $\mathrm{AE}(n=1108)$ & Non-AE $(n=2216)$ & STD \\
\hline Age (years) & $72.9 \pm 8.0$ & $71.6 \pm 8.0$ & 0.160 & $72.7 \pm 8.0$ & $72.5 \pm 7.8$ & 0.021 \\
\hline \multicolumn{7}{|l|}{ Comorbidity } \\
\hline Diabetes mellitus & $227(19.0)$ & $867(17.1)$ & 0.049 & $203(18.3)$ & $413(18.6)$ & -0.008 \\
\hline Hypertension & $596(49.9)$ & $2106(41.6)$ & 0.167 & $534(48.2)$ & $1075(48.5)$ & -0.006 \\
\hline Hyperlipidemia & $142(11.9)$ & $450(8.9)$ & 0.098 & $124(11.2)$ & $244(11.0)$ & 0.006 \\
\hline Chronic obstructive pulmonary disease & $229(19.2)$ & $657(13.0)$ & 0.169 & $191(17.2)$ & $361(16.3)$ & 0.025 \\
\hline Parkinsonism & $34(2.8)$ & $93(1.8)$ & 0.067 & $24(2.2)$ & $51(2.3)$ & -0.009 \\
\hline Ischemic heart disease & $236(19.8)$ & $791(15.6)$ & 0.108 & $196(17.7)$ & $405(18.3)$ & -0.015 \\
\hline Stroke & $165(13.8)$ & $408(8.1)$ & 0.185 & $123(11.1)$ & $252(11.4)$ & -0.009 \\
\hline Heart failure & $54(4.5)$ & $166(3.3)$ & 0.064 & $41(3.7)$ & $92(4.2)$ & -0.023 \\
\hline Alcoholism & $6(0.5)$ & $10(0.2)$ & 0.052 & $1(0.09)$ & $5(0.23)$ & -0.034 \\
\hline Drug abuse & $3(0.3)$ & $13(0.3)$ & -0.001 & $1(0.09)$ & $4(0.18)$ & -0.025 \\
\hline CCI score & $1.5 \pm 1.7$ & $1.2 \pm 1.5$ & 0.218 & $1.4 \pm 1.6$ & $1.4 \pm 1.7$ & 0.002 \\
\hline \multicolumn{7}{|l|}{ Tissue ablation } \\
\hline $5-15 \mathrm{~g}$ & 850 (71.2) & 3771 (74.5) & -0.075 & 791 (71.4) & $1576(71.1)$ & 0.006 \\
\hline $15-50 \mathrm{~g}$ & $288(24.1)$ & $1086(21.5)$ & 0.063 & $269(24.3)$ & $538(24.3)$ & 0.000 \\
\hline \multicolumn{7}{|l|}{ Urologic event in the previous three years } \\
\hline Urinary tract infection & $484(40.5)$ & $1192(23.6)$ & 0.370 & $401(36.2)$ & $790(35.6)$ & 0.011 \\
\hline Urinary retention & $495(41.5)$ & $1783(35.2)$ & 0.128 & $445(40.2)$ & $898(40.5)$ & -0.007 \\
\hline Bladder stone & $39(3.3)$ & 137 (2.7) & 0.033 & $34(3.1)$ & $67(3.0)$ & 0.003 \\
\hline \multicolumn{7}{|l|}{ Urologic drug use in the previous three months } \\
\hline Anti-muscarinic & $114(9.5)$ & $488(9.6)$ & -0.003 & $108(9.7)$ & $221(10.0)$ & -0.008 \\
\hline Alpha-blockers & $920(77.1)$ & $3817(75.4)$ & 0.038 & $851(76.8)$ & $1706(77.0)$ & -0.004 \\
\hline Propensity score & $0.235 \pm 0.105$ & $0.181 \pm 0.085$ & 0.569 & $0.216 \pm 0.082$ & $0.215 \pm 0.081$ & 0.008 \\
\hline Follow-up years & $6.3 \pm 3.8$ & $7.3 \pm 4.3$ & -0.225 & $6.5 \pm 3.9$ & $6.7 \pm 3.9$ & -0.050 \\
\hline
\end{tabular}

STD, standardized difference. BPH, benign prostatic hyperplasia. TURP, transurethral resection of the prostate. AE, adverse event. CCI, Charlson comorbidity index. 
The difference in the risk of fatal outcomes (e.g., all-cause mortality) between the AE and non-AE groups was evaluated using the Cox proportional hazard model. The difference in incidence of nonfatal outcomes (e.g., Transurethral Resection of Bladder Tumor (TUR-BT)) between the groups was evaluated using the Fine and Gray sub-distribution hazard model, which considers all-cause mortality as a competing risk. The study group (AE compared with non-AE groups) was the only explanatory variable in the survival analyses. The within-pair clustering of outcomes after propensity score matching was accounted for by using a robust standard error, known as a marginal model [19].

A two-sided $p$ of $<0.05$ was considered statistically significant, and no adjustment of multiple testing (multiplicity) was made in this study. All statistical analyses were performed using: Statistical Analysis System (SAS) (version 9.4, SAS Institute, Cary, NC, USA), including the procedures of "psmatch" for propensity score matching, "phreg" for survival analysis, and the macro "\% cif" for generating a cumulative incidence function under the Fine and Gray sub-distribution hazard method.

\section{Results}

\subsection{Study Population}

The data of 66,782 patients from the NHIRD were analyzed, as shown in Figure 1. Overall, 6254 patients who underwent TURP and 47,965 patients who underwent alpha blocker treatment were eligible for analysis. Both of the cohorts were further sub-grouped into an $\mathrm{AE}$ group and a non-AE group. After matching, we divided the included patients into the following four groups for analysis: TURP with AEs $(n=1108)$, TURP without AEs $(n=2216)$, alpha blockers with AEs $(n=2633)$, and alpha blockers without AEs $(n=5266)$. Tables 1 and 2 list the basic characteristics of the patients who underwent TURP and medication only, respectively. No significant difference (STD absolute value $<0.1$ ) was observed between the patients with AEs and those without AEs with respect to all of the covariates in either the TURP or medication-only groups.

\subsection{Association between AEs and Treatment Outcomes}

Table 3 details the relationship between the occurrence of post operation AEs and clinical outcomes during the follow-up period in the TURP group. Risk of all-cause mortality was comparable between the two groups (hazard ratio (HR): 1.07; 95\% CI: 0.93-1.23), as shown in Figure 2A. However, patients who had an AE within six months following operation had a risk of bladder cancer formation requiring TUR-BT that was subsequently increased when compared with patients without AE (sub-distribution hazard ratio (SHR): 2.3; 95\% CI: 1.56-3.39), as shown in Figure 2B. By contrast, the risk of prostate cancer formation remained similar between these groups (SHR: 1.20, 95\% CI: 0.83-1.74). The risk of postop long-term medication dependence, both for alpha blockers and antimuscarinic medications, was also significantly different between the two groups, as indicated in Figure 2C. In addition, patients with post-operation AE had higher risk of long-term dependence on medications for LUTS, hemorrhoids, and hip fractures caused by accidental falls during the first three-year follow-up period. 
Table 3. Treatment outcomes during follow-up of patients with BPH who underwent TURP.

\begin{tabular}{|c|c|c|c|c|}
\hline \multirow{2}{*}{ Variable } & \multirow{2}{*}{$\begin{array}{c}\mathrm{AE} \\
(n=1108)\end{array}$} & \multirow{2}{*}{$\begin{array}{l}\text { Non-AE } \\
(n=2216)\end{array}$} & \multicolumn{2}{|c|}{ AE vs. Non-AE } \\
\hline & & & HR/SHR (95\% CI) & $P$ \\
\hline \multicolumn{5}{|c|}{$\begin{array}{c}\text { Primary Outcome at the End of } \\
\text { Follow Up }\end{array}$} \\
\hline All-cause mortality & $431(38.9)$ & $827(37.3)$ & $1.07(0.93,1.23)$ & 0.332 \\
\hline Prostate cancer & $31(2.8)$ & $56(2.5)$ & $1.20(0.83,1.74)$ & 0.337 \\
\hline TUR-BT & $36(3.2)$ & $32(1.4)$ & $2.30(1.56,3.39)$ & $<0.001$ \\
\hline Radical cystectomy & $5(0.5)$ & $1(0.0)$ & NA & NA \\
\hline \multicolumn{5}{|c|}{$\begin{array}{l}\text { Secondary outcome during } \\
\text { three-year follow-up }\end{array}$} \\
\hline \multicolumn{5}{|c|}{ Medication dependence } \\
\hline Anti-muscarinic & $30(2.7)$ & $26(1.2)$ & $2.27(1.48,3.46)$ & $<0.001$ \\
\hline Alpha-blocker & $112(10.1)$ & $172(7.8)$ & $1.31(1.08,1.59)$ & 0.006 \\
\hline Inguinal hernia & $40(3.6)$ & $59(2.7)$ & $1.35(0.98,1.87)$ & 0.066 \\
\hline Hemorrhoids & $132(11.9)$ & $195(8.8)$ & $1.39(1.16,1.66)$ & $<0.001$ \\
\hline Stroke & $56(5.1)$ & $118(5.3)$ & $0.97(0.74,1.25)$ & 0.788 \\
\hline AMI & $8(0.7)$ & $19(0.9)$ & $0.80(0.40,1.57)$ & 0.511 \\
\hline Hip fracture & $21(1.9)$ & $20(0.9)$ & $2.26(1.37,3.71)$ & 0.001 \\
\hline
\end{tabular}

TURP, transurethral resection of the prostate. AE, adverse event. HR, hazard ratio. SHR, sub-distribution hazard ratio. CI, confidence interval. TUR-BT, transurethral resection of bladder tumor. NA, not applicable. AMI: Acute myocardial infarction.
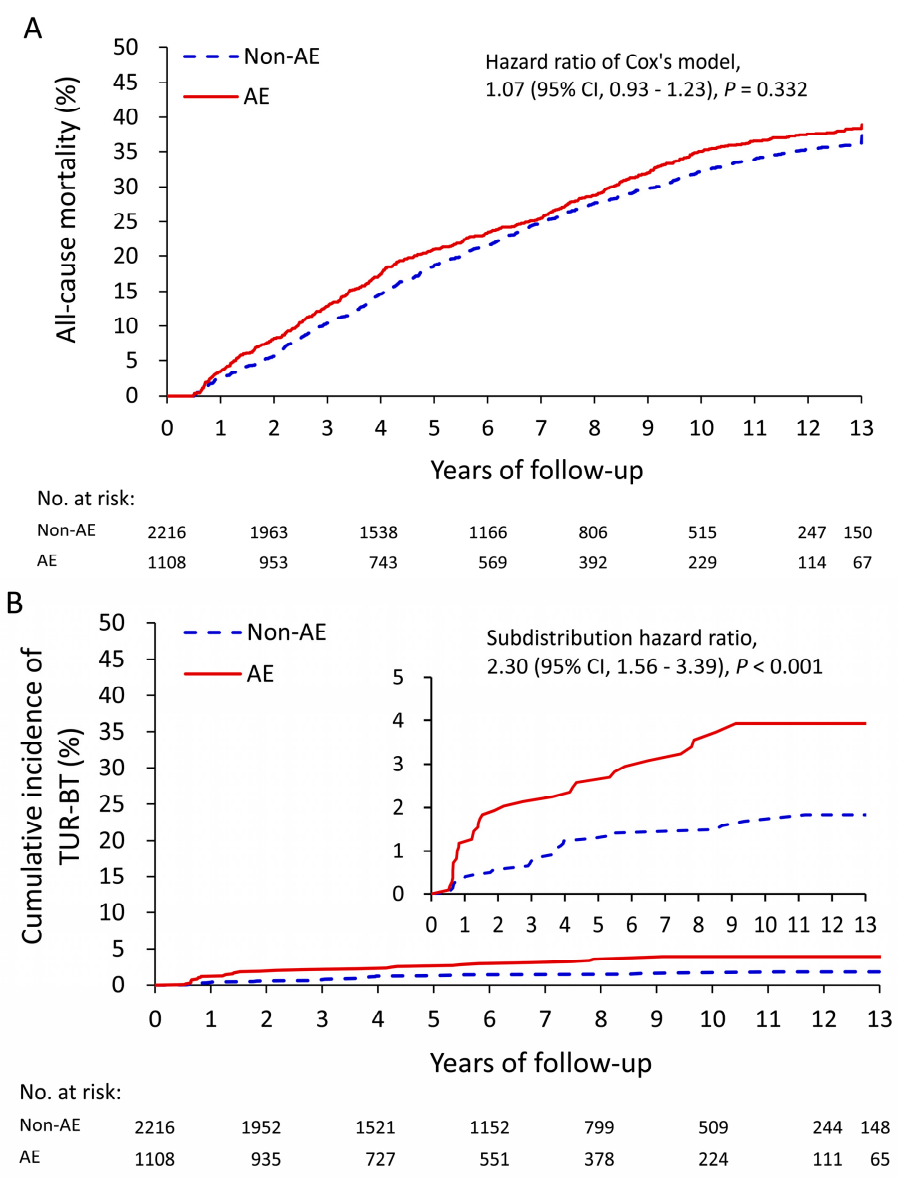

Figure 2. Cont. 


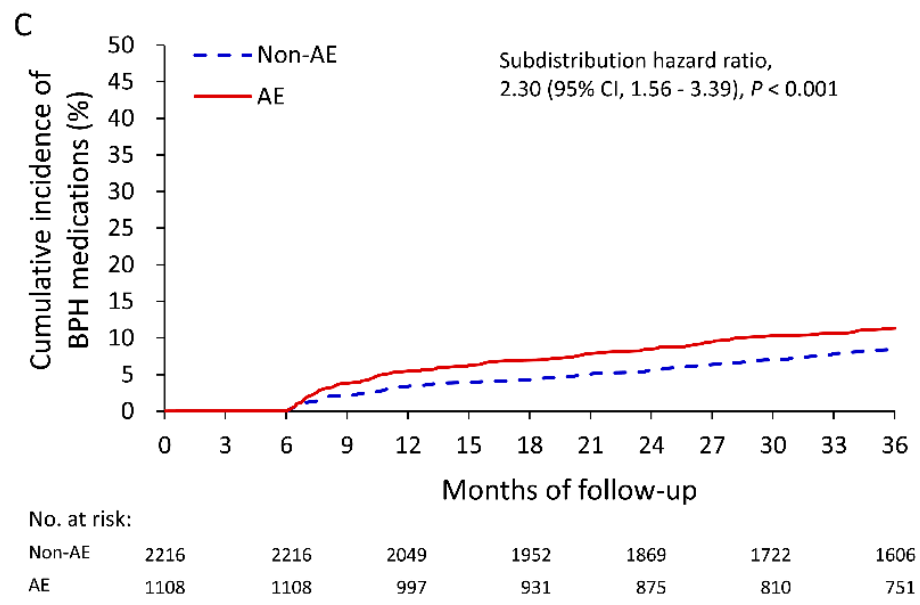

Figure 2. Unadjusted cumulative event rate of all-cause mortality (A), cumulative incidence of transurethral resection of the bladder tumor $(\mathbf{B})$, and long-term use of medications for treating benign prostatic hyperplasia $(\mathbf{C})$ in patients with and without adverse events during the first six-month follow-up in the TURP cohort.

Table 4 details the relationship between AEs following treatment with alpha blockers and clinical outcomes during the follow-up. Patients with AEs following alpha blocker treatment had a higher all-cause mortality rate (HR: 1.63, 95\% CI: 1.49-1.78) compared with those without AEs, as indicated in Figure 3A. Patients with AEs following alpha blocker treatment also had a higher risk of bladder cancer formation requiring TUR-BT or radical cystectomy, as shown in Figure 3B,C. By contrast, the risk of prostate cancer formation remained similar (sub-distribution hazard ratio (SHR): $1.16,95 \%$ CI: 0.84-1.61) between the two groups. In addition, patients with post-operation AEs had a higher risk of long-term dependence on medications for treating LUTS (Table 5).

Table 4. Treatment outcomes during follow-up of patients with BPH who received alpha blocker therapy.

\begin{tabular}{ccccc}
\hline & AE & Non-AE & \multicolumn{2}{c}{ AE vs. Non-AE } \\
\cline { 5 - 5 } Variable & $(\boldsymbol{n = 2 6 3 3 )}$ & $(\boldsymbol{n = 5 2 6 6 )})$ & HR/SHR (95\% CI) & $p$ \\
\hline Primary Outcome at the End of & & & & \\
Follow Up & & & & \\
\hline All-cause mortality & $1272(48.3)$ & $2060(39.1)$ & $1.63(1.49,1.78)$ & $<0.001$ \\
Prostate cancer & $44(1.7)$ & $89(1.7)$ & $1.16(0.84,1.61)$ & 0.354 \\
TUR-BT & $66(2.5)$ & $49(0.9)$ & $2.72(1.99,3.71)$ & $<0.001$ \\
Radical cystectomy & $15(0.6)$ & $3(0.1)$ & $7.68(2.54,23.28)$ & $<0.001$ \\
Secondary outcome during & & & & \\
three-year follow-up & & & & \\
Inguinal hernia & $55(2.1)$ & $96(1.8)$ & $1.12(0.85,1.47)$ & 0.418 \\
Hemorrhoids & $202(7.7)$ & $403(7.7)$ & $1.04(0.90,1.19)$ & 0.629 \\
Stroke & $143(5.4)$ & $262(5.0)$ & $1.06(0.90,1.26)$ & 0.475 \\
AMI & $30(1.1)$ & $45(0.9)$ & $1.33(0.91,1.92)$ & 0.137 \\
Hip fracture & $36(1.4)$ & $82(1.6)$ & $0.93(0.67,1.29)$ & 0.651 \\
\hline
\end{tabular}

$\overline{\mathrm{AE}}$, adverse event. HR, hazard ratio. SHR, sub-distribution hazard ratio. CI, confidence interval. TUR-BT, transurethral resection of the bladder tumor. AMI, acute myocardial infarction. 
A

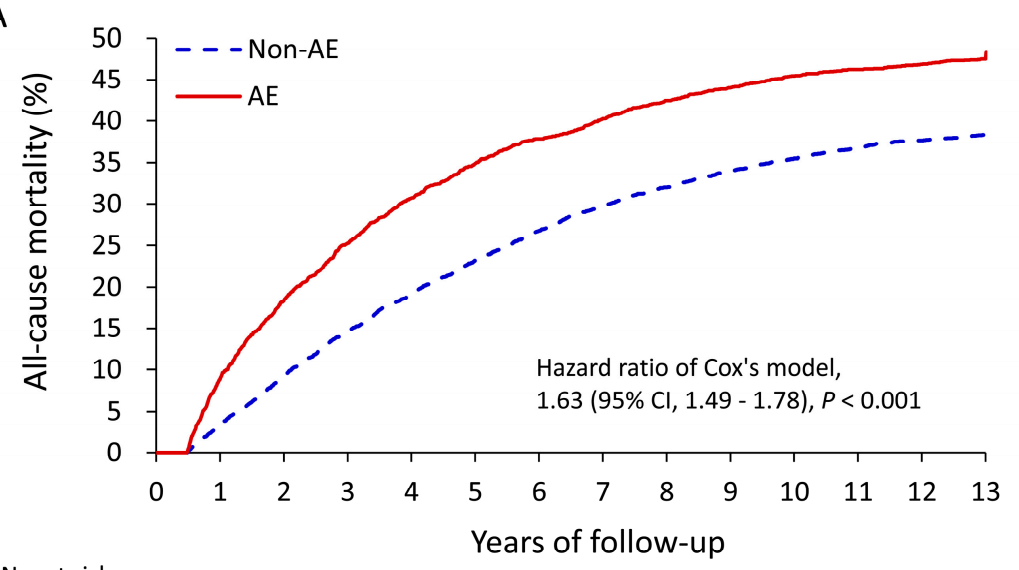

$\begin{array}{lrrrrrrrr}\text { No. at risk: } & & & & & & \\ \text { Non-AE } & 5266 & 4329 & 3196 & 2266 & 1490 & 929 & 481 & 304 \\ \text { AE } & 2633 & 1948 & 1339 & 945 & 620 & 348 & 185 & 101\end{array}$

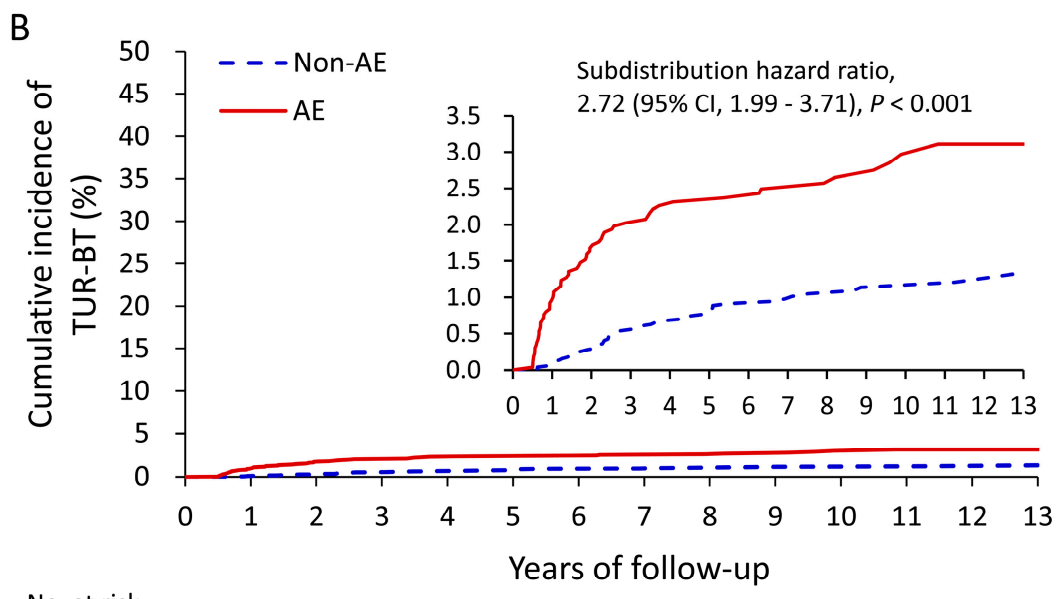

$\begin{array}{lrrrrrrrr}\text { No. at risk: } & & & & & & & \\ \text { Non-AE } & 5266 & 4317 & 3180 & 2248 & 1477 & 919 & 476 & 300 \\ \text { AE } & 2633 & 1912 & 1308 & 923 & 601 & 337 & 179 & 98\end{array}$

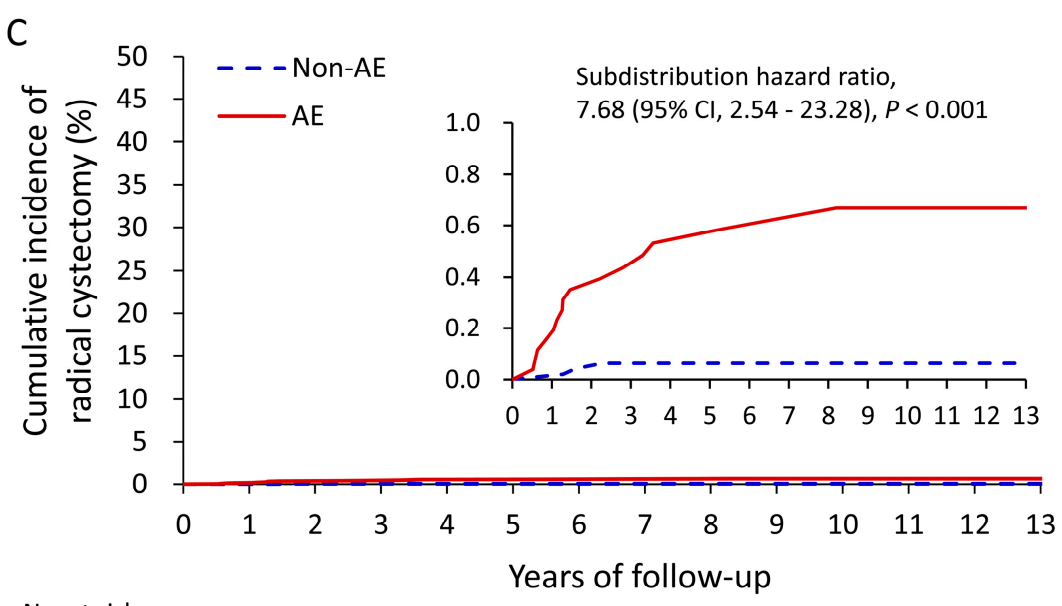

$\begin{array}{lrrrrrrrr}\text { No. at risk: } & & & & & & & \\ \text { Non-AE } & 5266 & 4327 & 3194 & 2265 & 1489 & 928 & 481 & 304 \\ \text { AE } & 2633 & 1941 & 1332 & 939 & 617 & 345 & 183 & 100\end{array}$

Figure 3. Unadjusted cumulative event rate of all-cause mortality (A), cumulative incidence of transurethral resection of bladder tumor (B), and cumulative incidence of radical cystectomy (C) in patients with and without adverse events during the first six-month follow-up in the medication-only cohort. 
Table 5. Association between baseline characteristics and long-term use of medications for treating $\mathrm{BPH}$ among patients with BPH who received TURP $(n=6254)$.

\begin{tabular}{cccc}
\hline Predictor & HR & 95\% CI & $p$ Value \\
\hline Age $\geq$ 75 years & 1.29 & $1.07-1.56$ & 0.008 \\
\hline BPH duration (years) & 1.13 & $1.10-1.16$ & $<0.001$ \\
\hline Adverse event during the six-month follow up & 1.45 & $1.18-1.79$ & $<0.001$ \\
\hline Hypertension & 1.82 & $1.49-2.22$ & $<0.001$ \\
\hline Hyperlipidemia & 1.51 & $1.17-1.94$ & 0.002 \\
\hline Urinary tract infection in the previous three years & 1.23 & $1.01-1.49$ & 0.039 \\
\hline NSAIDs use in the previous year & 1.35 & $1.11-1.63$ & 0.002 \\
\hline Alpha-blocker use in the previous three months & 1.61 & $1.21-2.14$ & 0.001
\end{tabular}

$\mathrm{BPH}$, benign prostatic hyperplasia. TURP, transurethral resection of the prostate. HR, hazard ratio. CI, confidence interval. NSAIDs, nonsteroidal anti-inflammatory drugs.

\section{Discussion}

UR, Urinary tract infection (UTI), gross hematuria with bladder tamponade, and bladder stone formation are all possible sequelae of benign prostate obstruction (BPO) and are regarded as absolute indications of TURP [20]. However, it is frustrating for both physicians and patients if the previously mentioned complications reoccur despite treatment, regardless of whether the treatment is in the form of a surgical or medical intervention. According to the literature, unfavorable events of TURP include UTI (1.7-8.2\%), UR (3-9\%), hematuria with clot retention (2-5\%), urethral strictures (2.2-9.8\%), and bladder neck contractures (0.3-9.2\%) [21]. The incidence of unfavorable events following treatment with alpha blockers varies among reports [22]. Nevertheless, no studies have focused on whether these unfavorable events affect older adults during long-term follow-up.

Before comparing the clinical outcomes of the four cohorts, 1:1 propensity score matching [23] was performed to ensure that the characteristics of both groups were similar and that more objective data could be obtained. Therefore, as detailed in Tables 2 and 6, the distribution of parameters, including age, incidence of comorbidities, and Charlson comorbidity index, did not differ significantly between each two groups. The major finding of this research is that post-treatment AEs (whether following surgical or medical intervention) are significantly correlated with bladder cancer formation during lifelong follow-up. Bladder cancer is the ninth most common cancer worldwide, with an estimated 430,000 new cases in 2012 [24]. Bladder cancer resulted in 170,000 deaths globally in 2011, which demonstrates an increase from the 114,000 deaths in 1990 of $19.4 \%$ after adjusting for the increase in the total world population [25]. Data collected using the Taiwan Cancer Registry database suggest that the incidence of bladder cancer in the general population is approximately $0.21 \%$ [26]. However, the patients included in our study exhibited a higher bladder cancer incidence compared with the Taiwan general population. Patients with post-treatment AE even had a greater risk of bladder cancer than those without AE. This phenomenon indicates that an unfavorable treatment outcome for BPO is associated with an increased incidence of bladder cancer. 
Table 6. Baseline characteristics of patients with BPH who received alpha blocker therapy grouped according to the presence or absence of AEs during the six-month follow-up period.

\begin{tabular}{|c|c|c|c|c|c|c|}
\hline \multirow[b]{2}{*}{ Variable } & \multicolumn{3}{|c|}{ Before Matching } & \multicolumn{3}{|c|}{ After Matching } \\
\hline & $\mathrm{AE}(n=2743)$ & Non-AE $(n=45,222)$ & STD & $\mathrm{AE}(n=2633)$ & Non-AE $(n=5266)$ & STD \\
\hline Age (years) & $70.1 \pm 10.3$ & $66.7 \pm 9.9$ & 0.335 & $69.8 \pm 10.3$ & $70.1 \pm 10.8$ & -0.023 \\
\hline \multicolumn{7}{|l|}{ Comorbidity } \\
\hline Diabetes mellitus & $641(23.4)$ & $7414(16.4)$ & 0.175 & $589(22.4)$ & $1193(22.7)$ & -0.007 \\
\hline Hypertension & $1264(46.1)$ & $17292(38.2)$ & 0.159 & $1192(45.3)$ & $2424(46.0)$ & -0.015 \\
\hline Hyperlipidemia & $309(11.3)$ & $5598(12.4)$ & -0.035 & $300(11.4)$ & $604(11.5)$ & -0.002 \\
\hline Chronic obstructive pulmonary disease & $466(17.0)$ & $4752(10.5)$ & 0.189 & $412(15.6)$ & $853(16.2)$ & -0.015 \\
\hline Parkinsonism & $85(3.1)$ & $539(1.2)$ & 0.132 & $75(2.8)$ & $155(2.9)$ & -0.006 \\
\hline Chronic kidney disease & $324(11.8)$ & $3010(6.7)$ & 0.179 & $296(11.2)$ & $603(11.5)$ & -0.007 \\
\hline Ischemic heart disease & $484(17.6)$ & 5985 (13.2) & 0.122 & $450(17.1)$ & $936(17.8)$ & -0.018 \\
\hline Stroke & $533(19.4)$ & $3580(7.9)$ & 0.340 & $460(17.5)$ & $934(17.7)$ & -0.007 \\
\hline Heart failure & $173(6.3)$ & $1067(2.4)$ & 0.195 & $143(5.4)$ & $312(5.9)$ & -0.021 \\
\hline Alcoholism & $33(1.2)$ & $347(0.8)$ & 0.044 & $32(1.22)$ & $74(1.41)$ & -0.017 \\
\hline Drug abuse & $19(0.7)$ & $238(0.5)$ & 0.021 & $19(0.72)$ & $40(0.76)$ & -0.004 \\
\hline CCI score & $1.6 \pm 1.9$ & $1.0 \pm 1.5$ & 0.385 & $1.5 \pm 1.8$ & $1.6 \pm 1.9$ & -0.021 \\
\hline \multicolumn{7}{|l|}{ Urology event in the previous three years } \\
\hline Urinary tract infection & $893(32.6)$ & $4408(9.7)$ & 0.582 & $783(29.7)$ & $1541(29.3)$ & 0.010 \\
\hline Urinary retention & $529(19.3)$ & $1693(3.7)$ & 0.502 & 419 (15.9) & $780(14.8)$ & 0.031 \\
\hline Bladder stone & $39(1.4)$ & $223(0.5)$ & 0.095 & $32(1.2)$ & $52(1.0)$ & 0.022 \\
\hline \multicolumn{7}{|l|}{ Urologic drug use in the previous three months } \\
\hline Anti-muscarinic & $79(2.9)$ & $675(1.5)$ & 0.095 & $74(2.8)$ & $162(3.1)$ & -0.016 \\
\hline Alpha-blockers & - & - & - & - & - & - \\
\hline Propensity score & $0.121 \pm 0.126$ & $0.053 \pm 0.053$ & 0.705 & $0.105 \pm 0.096$ & $0.104 \pm 0.095$ & 0.003 \\
\hline Follow-up years & $5.1 \pm 3.8$ & $6.7 \pm 4.3$ & -0.404 & $5.1 \pm 3.8$ & $5.9 \pm 3.9$ & -0.200 \\
\hline
\end{tabular}

STD, standardized difference. AE, adverse event. CCI, Charlson comorbidity index. 
Multiple factors are associated with bladder cancer development. However, smoking tobacco is the main known contributor [27]. The number of cigarettes smoked, degree of inhalation, type of tobacco, use of filters, and smoking cessation all have significant relationships with bladder cancer development [28]. In addition, common candidate genes or pathways, such as carcinogenic metabolizing genes, DNA repair genes, apoptosis-related genes, and microRNA-related genes, have been studied widely and can contribute to the risk of bladder cancer [29]. Clinically, men with severe LUTS have a $64 \%$ higher relative risk of bladder cancer compared with men who report no LUTS. A recent study even revealed that patients with $\mathrm{BPH}$ who underwent TURP were at a higher risk of bladder cancer $(\mathrm{HR}=6.17,95 \%, \mathrm{CI}=3.68-10.3)$ than those who did not [30]. The sequelae of unfavorable BPO treatment outcomes, such as high post-voiding residual urine volume, repeat UTIs, chronic bladder inflammation, and chronic UR, all increase urothelial exposure to carcinogens and, thus, increase the risk of bladder cancer [31]. After chronic inflammation develops, it can mediate cancer pathogenesis by stimulating malignant cell growth, invasion, and metastasis through the recruitment of inflammatory cells and signaling molecules [32]. Therefore, an unfavorable treatment outcome for $\mathrm{BPO}$ is associated with an increasing bladder cancer incidence.

Similar to bladder cancer development, that of prostate cancer is positively associated with chronic tissue inflammation and bacterial infection [33]. Another study also reported that chronic tissue inflammation is associated with an increased risk of both prostate and bladder cancer, and a subgroup analysis by ethnicity suggested that the association was much stronger in Asian patients than in Caucasian patients [34]. A study pertaining to microbiomes indicated a prevalence of pro-inflammatory bacteria and uro-pathogens in the urinary tract of men with prostate cancer [35]. Urinary microbiomes influence chronic inflammation in the prostate and lead to prostate cancer development and progression [35]. Nevertheless, the findings of our study suggest that an unfavorable treatment outcome for BPO is not associated with prostate cancer development. Our explanation for this phenomenon is that prostate cancer development is mediated in part by chronic inflammation as well as by genetics and exposure to toxins in the environment. Thus, compared with unfavorable BPO treatment outcomes, other factors such as age, race, family medical history, lifestyle, and presence of metabolic syndrome might be stronger contributors to prostate cancer development.

Another notable finding of this study is that the treatment outcome of BPO had a substantial impact on the health status of male patients. In the medication-only group, patients with AEs, despite regular alpha blocker treatment, had a higher risk of all-cause mortality. By contrast, patients with $\mathrm{AE}$ following TURP also had increased risk of hemorrhoids, hip fracture caused by accidental falls, and medication dependence (alpha blockers or antimuscarinic medications) during a three-year follow-up. Many patients have persistent voiding dysfunction after surgical treatment for BPO. Older age, history of diabetes, history of cerebrovascular accidents, and preoperative antimuscarinic drug use are possible risk factors of continuing medical therapy following TURP [36]. We used 1:1 propensity score matching to eliminate the influence of the previously mentioned factors and concluded that the occurrence of AE within the six months following operation is also strongly associated with lifelong dependence on urologic medications.

This study has some limitations as a result of the NHIRD data structure. First, this database does not provide detailed personal information such as family cancer history, laboratory parameters, cigarette use, environmental toxin exposure, body mass index (BMI), and dietary habits, which are all confounding variables that influence prostate and bladder cancer development. Second, we used a strict criterion to divide our study population into two categories: patients with or without AEs. Thus, we could not assess how the duration, frequency, and severity of AEs affected treatment outcomes. Third, the use of laser prostatic vaporization or vapor-resection, which is not reimbursed by the Taiwan NHI, has only become increasingly common in the last decade [37]. Thus, patients receiving prostate laser treatment were not included in this database. However, despite these limitations, we believe that this is innovative and valid research. This study confirmed that unfavorable treatment outcomes of $\mathrm{BPH}$ increase the incidence of bladder cancer and have negative health effects among older men. 


\section{Conclusions}

This nationwide database study reveals that unfavorable outcomes of BPH following either medication-based or surgical treatment are associated with a higher bladder cancer incidence. These outcomes, subsequently, lead to negative health effects in older men.

Author Contributions: Conception and design: Y.-H.L. and C.-P.H.; enrollment of patients and acquisition of data: K.-H.T., Y.-H.L., T.-H.C., C.-L.C., and P.-L.C.; drafting of the manuscript: C.-P.H., Y.-H.L., T.-H.C. and H.-H.J.; statistical analysis: C.-L.C. and H.-H.J.; analysis and interpretation of data: K.-H.T., Y.-H.L., C.-P.H. and C.-L.C.; supervision: K.-H.T. We confirm that all authors read and approved the final manuscript.

Funding: The Chang Gung Memorial Hospital (Grant numbers: CRPG3A1061, CMRPG3A1062, CMRPG3E1071, CMRPG3G0821, CGRPG2F0011) and the National Science Council, Taiwan (NSC 104-2314-B-182A-140-MY3) supported this work.

Conflicts of Interest: The authors declare that they have no competing interests.

\section{References}

1. Verhamme, K.M.C.; Dieleman, J.P.; Bleumink, G.S.; van der Lei, J.; Sturkenboom, M.C.J.M.; Triumph Pan European Expert Panel. Incidence and prevalence of lower urinary tract symptoms suggestive of benign prostatic hyperplasia in primary care-the Triumph project. Eur. Urol. 2002, 42, 323-328. [CrossRef]

2. Berry, S.J.; Coffey, D.S.; Walsh, P.C.; Ewing, L.L. The development of human benign prostatic hyperplasia with age. J. Urol. 1984, 132, 474-479. [CrossRef]

3. Irwin, D.E.; Kopp, Z.S.; Agatep, B.; Milsom, I.; Abrams, P. Worldwide prevalence estimates of lower urinary tract symptoms, overactive bladder, urinary incontinence and bladder outlet obstruction. BJU Int. 2011, 108, 1132-1138. [CrossRef] [PubMed]

4. Jacobsen, S.J.; Jacobson, D.J.; Girman, C.J.; Ewing, L.L. Natural history of prostatism: Risk factors for acute urinary retention. J. Urol. 1997, 158, 481-487. [CrossRef]

5. Perchon, L.F.; Pintarelli, V.L.; Bezerra, E.; Thiel, M.; Dambros, M. Quality of life in elderly men with aging symptoms and lower urinary tract symptoms (LUTS). Neurourol. Urodyn. 2011, 30, 515-519. [CrossRef] [PubMed]

6. Oesterling, J.E. Benign prostatic hyperplasia: A review of its histogenesis and natural history. prostate. 1996, 29, 67-73. [CrossRef]

7. Saigal, C.S.; Joyce, G. Economic costs of benign prostatic hyperplasia in the private sector. J. Urol. 2005, 173, 1309-1313. [CrossRef]

8. Nickel, J.C.; Méndez-Probst, C.E.; Whelan, T.F.; Paterson, R.F.; Razvi, H. 2010 Update: Guidelines for the management of benign prostatic hyperplasia. Can. Urol. Assoc. J. 2010, 4, 310. [CrossRef] [PubMed]

9. Fusco, F.; Palmieri, A.; Ficarra, V.; Giannarini, G.; Novara, G.; Longo, N.; Verze, P.; Creta, M.; Mirone, V. $\alpha 1$-blockers improve benign prostatic obstruction in men with lower urinary tract symptoms: A systematic review and meta-analysis of urodynamic studies. Eur. Urol. 2016, 69, 1091-1101. [CrossRef]

10. Mayer, E.K.; Kroeze, S.G.; Chopra, S.; Bottle, A.; Patel, A. Examining the 'gold standard': A comparative critical analysis of three consecutive decades of monopolar transurethral resection of the prostate (TURP) outcomes. BJU Int. 2012, 110, 1595-1601. [CrossRef]

11. Lin, Y.H.; Hou, C.P.; Chen, T.H.; Juang, H.H.; Chang, P.L.; Yang, P.S.; Tsui, K.H. Transurethral resection of the prostate provides more favorable clinical outcomes compared with conservative medical treatment in patients with urinary retention caused by benign prostatic obstruction. BMC Geriatr. 2018. [CrossRef] [PubMed]

12. Hou, C.P.; Lin, Y.H.; Chen, T.H.; Chang, P.L.; Juang, H.H.; Chen, C.L.; Tsui, K.H. Transurethral resection of the prostate achieves favorable outcomes in stroke patients with symptomatic benign prostate hyperplasia. Aging Male 2018, 21, 9-16. [CrossRef] [PubMed]

13. Lin, Y.H.; Hou, C.P.; Chen, T.H.; Juang, H.H.; Chang, P.L.; Yang, P.S.; Tsui, K.H. Is diabetes mellitus associated with clinical outcomes in aging males treated with transurethral resection of prostate for bladder outlet obstruction: Implications from Taiwan Nationwide population-based cohort study. Clin. Interv. Aging 2017. [CrossRef] [PubMed] 
14. Hsiao, F.Y.; Yang, C.L.; Huang, Y.T.; Huang, W.F. Using Taiwan's National Health Insurance Research Databases for Pharmacoepidemiology Research. J. Food. Drug Anal. 2007, 15, 99-108.

15. Charlson, M.E.; Pompei, P.; Ales, K.L.; McKenzie, C.R. A new method of classifying prognostic comorbidity in longitudinal studies: Development and validation. J. Chron. Dis. 1987, 40, 373-383. [CrossRef]

16. Wu, C.S.; Lai, M.S.; Gau, S.S.; Wang, S.C.; Tsai, H.J. Concordance between patient self-reports and claims data on clinical diagnoses, medication use, and health system utilization in Taiwan. PLoS ONE 2014. [CrossRef] [PubMed]

17. Wu, C.-Y.; Chen, Y.-J.; Ho, H.-J.; Hsu, Y.-C.; Kuo, K.N.; Wu, M.-S.; Lin, J.-T. Association between nucleoside analogues and risk of hepatitis $\mathrm{B}$ virus-related hepatocellular carcinoma recurrence following liver resection. JAMA 2012, 308, 1906-1913. [CrossRef] [PubMed]

18. Chen, D.Y.; See, L.C.; Liu, J.R.; Chuang, C.K.; Pang, S.T.; Hsieh, I.C.; Wen, M.S.; Chen, T.H.; Lin, Y.C.; Liaw, C.C.; et al. Risk of Cardiovascular Ischemic Events After Surgical Castration and Gonadotropin-Releasing Hormone Agonist Therapy for Prostate Cancer: A Nationwide Cohort Study. J. Clin. Oncol. 2017, 35, 3697-3705. [CrossRef]

19. Austin, P.C.; Fine, J.P. Propensity-score matching with competing risks in survival analysis. Stat. Med. 2019, 38, 751-777. [CrossRef]

20. Davidian, M.H. Guidelines for the treatment of benign prostatic hyperplasia. US Pharm. 2016, 41, 36-40.

21. Rassweiler, J.; Teber, D.; Kuntz, R.; Hofmann, R. Complications of transurethral resection of the prostate (TURP)—incidence, management, and prevention. Eur. Urol 2006, 50, 969-980. [CrossRef] [PubMed]

22. Lepor, H. Alpha blockers for the treatment of benign prostatic hyperplasia. Rev. Urol. 2007, 9, 181-190. [CrossRef]

23. Caliendo, M.; Kopeinig, S. Some practical guidance for the implementation of propensity score matching. J. Econ. Surv. 2008, 22, 31-72. [CrossRef]

24. Ferlay, J.; Soerjomataram, I.; Ervik, M.; Dikshit, R.; Eser, S.; Mathers, C.; Rebelo, M.; Parkin, D.M.; Forman, D.; Bray, F. GLOBOCAN 2012 v1.0, cancer incidence and mortality worldwide. 2013, IARC CancerBase No. 11. Lyon, France: International Agency for Research on Cancer. Available online: http://globocan.iarc.fr. (accessed on 12 December 2013).

25. Lozano, R.; Naghavi, M.; Foreman, K.; Lim, S.; Shibuya, K.; Aboyans, V.; Abraham, J.; Adair, T.; Aggarwal, R.; Ahn, S.Y.; et al. Global and regional mortality from 235 causes of death for 20 age groups in 1990 and 2010: A systematic analysis for the Global Burden of Disease Study 2010. Lancet 2012, 9859, 2095-2128. [CrossRef]

26. Chiang, C.J.; Lo, W.C.; Yang, Y.W.; You, S.L.; Chen, C.J.; Lai, M.S. Incidence and survival of adult cancer patients in Taiwan, 2002-2012. J. Formos. Med. Assoc. 2016, 115, 1076-1088. [CrossRef] [PubMed]

27. Rink, M.; Furberg, H.; Zabor, E.C.; Xylinas, E.; Babjuk, M.; Pycha, A.; Montorsi, F. Impact of smoking and smoking cessation on oncologic outcomes in primary non-muscle-invasive bladder cancer. Eur. Urol. 2013, 63, 724-732. [CrossRef]

28. Vincis, P.; Esteve, J.; Hartge, P.; Hoover, R.; Silverman, D.T.; Terracini, B. Effects of timing and type of tobacco in cigarette-induced bladder cancer. Cancer Res. 1998, 48, 3849-3852.

29. Chu, H.; Wang, M.; Zhang, Z. Bladder cancer epidemiology and genetic susceptibility. J. Biomed. Res. 2013, 27, 170 .

30. Fang, C.W.; Liao, C.H.; Wu, S.C.; Muo, C.H. Association of benign prostatic hyperplasia and subsequent risk of bladder cancer: An Asian population cohort study. World J. Urol. 2018, 36, 931-938. [CrossRef]

31. Burger, M.; Catto, J.W.; Dalbagni, G.; Grossman, H.B.; Herr, H.; Karakiewicz, P.; Lotan, Y. Epidemiology and risk factors of urothelial bladder cancer. Eur. Urol. 2013, 63, 234-241. [CrossRef]

32. Nesi, G.; Nobili, S.; Cai, T.; Caini, S.; Santi, R. Chronic inflammation in urothelial bladder cancer. Virchows Archiv 2015, 467, 623-633. [CrossRef]

33. Platz, E.A.; Kulac, I.; Barber, J.R.; Drake, C.G.; Joshu, C.E.; Nelson, W.G.; Thompson, I.M. A prospective study of chronic inflammation in benign prostate tissue and risk of prostate cancer: Linked PCPT and SELECT cohorts. Cancer Epidemiol. Prev. Biomark. 2017, 26, 1549-1557. [CrossRef]

34. Dai, X.; Fang, X.; Ma, Y.; Xianyu, J. Benign prostatic hyperplasia and the risk of prostate cancer and bladder cancer: A meta-analysis of observational studies. Medicine 2016. [CrossRef] [PubMed]

35. Shrestha, E.; White, J.R.; Yu, S.H.; Kulac, I.; Ertunc, O.; De Marzo, A.M.; Sfanos, K.S. Profiling the urinary microbiome in men with positive versus negative biopsies for prostate cancer. J. Urol. 2018, 199, 161-171. [CrossRef] 
36. Han, H.H.; Ko, W.J.; Yoo, T.K.; Oh, T.H.; Kim, D.Y.; Kwon, D.D.; Jung, T.Y. Factors associated with continuing medical therapy after transurethral resection of prostate. Urology 2014, 84, 675-680. [CrossRef]

37. Lee, W.C.; Lin, Y.H.; Hou, C.P.; Chang, P.L.; Tsui, K.H. Prostatectomy using different lasers for the treatment of benign prostate hyperplasia in aging males. Clin. Interv. Aging 2013, 8, 1483-1488.

(C) 2019 by the authors. Licensee MDPI, Basel, Switzerland. This article is an open access article distributed under the terms and conditions of the Creative Commons Attribution (CC BY) license (http://creativecommons.org/licenses/by/4.0/). 